\title{
Molecular targets to develop future antimicrobials
}

\author{
MOHAMMED Z. AL-KHAYYAT \\ University of Mosul, Biology Department, Mosul, Iraq
}

\begin{abstract}
To overcome antibiotic resistance in pathogenic microorganisms, research work is being directed towards finding alternative targets in bacteria towards which novel antibiotics can be designed. One of the targets is cell-to-cell communication pathway, also called quorum sensing which is promising since it controls the expression of virulence genes. Another possible target is the segregation apparatus that is present in low copy number plasmids that may contain antibiotic resistance genes. Cellular elements that function in division or maintain the shape of the micro-organisms may be also used as alternative targets to affect cytokinesis or cause abnormalities in shape, which can or may lead to cell lysis. Additionally, certain metabolic pathways present in microbes do not exist in mammals and, therefore, may be exploited as novel targets without affecting the human host. Screening of ligands or chemical compounds from natural products may be useful in finding inhibitors acting on the above-mentioned cellular components, and it may lead to the design of future antibiotics.
\end{abstract}

Key words: antimicrobials, quorum sensing, cytoskeleton, shikimate, isoprenoid

\section{Introduction}

The widespread occurrence of antibiotic resistance in developing countries is due to several reasons, but mainly due to the use of antibiotics without prescription in countries like Sudan, Brazil, and Bangladesh (Morgan et al., 2011). Among poor people, sharing of prescribed antibiotics with other patients, not completing the course of treatment and obtaining drugs from untrustworthy drug sellers or suppliers also contribute to the increase in antibiotic resistance. In a study conducted in Vietnam on ciprofloxacin, an antibiotic, it was found that only $20 \mathrm{mg}$ was present in a $500 \mathrm{mg}$ capsule, illustrating the extent of the problem connected to untrustworthy drug supplies (Hart and Kariuki, 1998).

Micro-organisms develop resistance to antibiotics by various mechanisms. Staphylococcus aureus produces the enzyme pencillinase that destroys the $\beta$-lactam ring present in penicillin, inhibiting cell wall synthesis (Bush, 2013). Pathogens can add chemical modifications to antibiotics, e.g., perform acetylation of chloramphenicol or phosphorylate aminoglycosides that target the synthesis of proteins at the ribosome level, which renders them inactive as seen in the case of Providencia stuartii, Enterococcus faecium, and Serratia marcescens (Ramirez and Tolmasky, 2010; Wilson, 2014). Bacteria can also develop efflux systems capable of pumping antibiotics out of the cells like that found in Escherichia coli, which pumps tetracycline out (Piddock, 2006). Mutations in bacterial genomes are yet another way of gaining antibiotic resistance; e.g., point mutations in the $r p o B$ gene of $E$. coli confers resistance to rifamycin, an antibiotic acting by inhibiting DNA-dependent RNA polymerase (Goldstein, 2014). A study of bacteria isolated from burns and wounds of patients from Iraq showed that 64 isolates of Pseudomonas aeruginosa were 100\% resistant to Aztreonam, piperacillin, carpenicillin, cefepime, cefixime, cephalexin, ceftriaxone, cefotixime, ceftazidime, trimethoprim, and co-trimoxazole (Hussein et al., 2017). In India, a study of 922 pathogenic bacteria (Pseudomonas spp., Aceinetobacter spp., Klebsiella spp., E. coli, and Enterobacter spp.) was conducted in the tertiary trauma care center where the antibiotic resistance rates were measured. The isolates were resistant to carbapenems, aminoglycosides, flouroquinolones,

* Corresponding author: University of Mosul, Biology Department, Mosul, Iraq; e-mail: mzsaeed19@hotmail.com 
third generation cephalosporins, $\beta$ lactam $\beta$-lactamase inhibitor combinations in percentages of 50,66, 76, 88 and $63 \%$, respectively (Behera and Mathur, 2011).

Many approaches are currently used to find new targets for which new antibiotics are developed. Polyprodrug antimicrobials were suggested as new antibiotics (e.g., triclosan repeated units) for methicilin resistant $S$. aureus. These act by damaging the bacterial cell membrane (Cao et al., 2018). Culp and Wright (2017) suggested bacterial proteases as molecular targets since proteases have important roles in bacterial biochemistry and physiology. This review outlines new molecular targets for which antibiotics could be developed to eliminate microbes resistant to currently available drugs.

\section{Quorum sensing as an antivirulence target}

Bacteria can respond to environmental stimuli by releasing molecules called autoinducers in a cellular signaling pathway referred to as quorum sensing. When cell density of microorganisms increases, signal molecules also increase in concentration as a function of cell density, and initiate the transcription of target genes, enabling the microorganisms to adapt to the environment (Miller and Bassler, 2001). The quorum sensing system was first described in the bioluminescent Vibrio fischeri, a marine bacterium which lives in a symbiotic relationship with marine fish and squids producing light. Bioluminescence is facilitated by the action of two regulatory proteins LuxI and LuxR on luciferase structural genes (Fuqua and Greenberg, 2002).

Four basic types of quorum sensing have been described: a) LuxI/LuxR homologs, b) the LuxS/Autoinducer-2 system, c) catecholamines as signal molecules, as in Salmonella and Shigella species, and d) accessory gene regulator as in S. aureus (Asad and Opal, 2008). In the Serratia species, the LuxI/R homolog quorum sensing system is present; $\mathrm{SprI} / \mathrm{SprR}$ account for the production of lipase, nuclease, chitinase. and protease (Van Houdt and Michiels, 2005). In S. marcescens, another homolog system, SmaI/SmaR is responsible for bacterial motility, haemolytic activity, biofilm formation, synthesis of carbapenem, prodigiosin, caseinase, and chitinase (Thomson et al., 2000; Coulthurst et al., 2006). Pectobacterium carotovorum, a plant pathogen causing soft rot diesease, uses 3-oxo-hexanoyl homoserine lactone, and 3-oxo-octanoyl homoserine lactone as LuxI/LuxR homologs to control the secretion of cell wall tissue cellulases, proteases, and pectin lysis enzymes to damage crops and vegetables (Barnard and Salmond, 2007; Liu et al., 2008). Joshi et al. (2016) suggested that eugenol and carvacrol can interfere with the quorum sensing system, ExpI/ ExpR in pectobacteria. These two phenolic compounds caused a substantial decrease in biofilm formation and inhibited the activity of tissue degrading enzymes, pectate lyase, protease, and polygalacturonase.

Much research was carried out (reviewed in detail by Moghaddam et al., 2014) to find inhibitors that act on the complicated circuit of quorum sensing present in $P$. aeruginosa which contains more than one LuxI/R homolog with positive and negative regulators. Extracts of Panax ginensing eliminated pneumonia in tested animals caused by $P$. aeruginosa via its effects on virulence factors since it is involved in the reduction of the production of autoinducers LasA and LasB (Song et al., 2010). Vandeputte et al. (2011) analyzed the activity of various flavonoids on the quorum sensing of $P$. aeruginosa. This study has shown that: a) naringenin, taxifolin, and eriodicytol decreased both, the pyocyanin production and the activity of elastase, b) the expression of lasI, lasR, rhlI, rhlR, las $B$, rhlA, and phzA1 genes was affected by both taxifolin and naringenin, $c$ ) naringenin inhibited the synthesis of the autoinducers of both systems LasI/R and RhlI/R, d) lasI and rhlI deficient mutants had a malfunctioning RhlR-inducer complex as well as low level of inducer synthesis.

\section{Plasmid partition systems}

Plasmids are extrachromosomal DNA molecules present in microbes which code for certain genes like antibiotic resistance genes and, in certain cases, virulence genes (Austin and Sengupta, 2011). Therefore, plasmids may serve as potential targets for developing antibiotics. Low copy number plasmids rely on partition systems to ensure that each new daughter cell of the bacterium receives a copy of a plasmid molecule (Baxter and Funnell, 2014). The plasmid partition system is composed of a centromere-like site and two proteins. The first protein is the DNA binding molecule (designated ParB) which binds to the centromeric site and acts where the segregation apparatus is formed; the second protein, usually designated ParA, has an ATPase or GTPase activity and provides energy for plasmid molecules to be located at 
the opposite sides of the cell (Ebersbach and Gerdes, 2005; Baxter and Funnell, 2014).

Partition systems or the segregation apparatus can be divided into three types. Type I system is characterized by the presence of Walker ATPases, such as those of the virulence plasmids, e.g., pWR501 of Shigella flexneri (Sergueev et al., 2005). Type II system contains a protein with an actin-like fold, possessing ATPase activity such as that found in plasmid R1 isolated from E. coli (Salje et al., 2010). Type III system contains proteins with tublin-like fold, possessing GTPase activity (Larsen et al., 2007). Type I segregation systems can be subcategorized into two types, designated Ia and Ib. Type Ia ParA and ParB proteins were shown to be composed of 321-420 and 312-342 amino acid residues, respectively, whereas type Ib ParA and ParB possess 192-308 and 46-131 amino acids, respectively. Type Ia ParA proteins have both segregation and transcription autoregulation of their partition operons; hence, the helix-turn-helix DNA-binding domain is located at the N-terminal and composed of 108-130 amino acid residues. In contrast, the helixturn-helix DNA-binding domain is present in ParB protein of type Ib and, therefore, functions as a transcriptional regulator. The location of the centromere in the type Ia segregation system is downstream of the operon, where as it is upstream in type Ib (Gerdes et al., 2000).

Streptococcus pyogenes contains the Ib partition system present on pSM19035 plasmid (Ceglowski et al., 1993). This system is composed of Delta 2, a ParA analog possessing Walker ATPase, and since it is as dimer, it has been designated as $\delta 2$. The centromere binding protein, which is also a dimer, called Omega 2 and designated $\omega 2$, binds two sets of three parS centromeric sites. The $\delta$ monomer structure has been shown to contain eight $\beta$-strands surrounded by twelve $\alpha$-helices (Ceglowski et al., 1993; Pratto et al., 2008). Omega $\left(\omega_{2}\right)$ controls the partition system by binding to promoter regions upstream of $\operatorname{cop} S$, delta and omega genes. These regulatory regions have the $5^{\prime}$-WATCACW- 3 ' sequence organized as 10 , seven and nine times repeated hepatads, with deferent directions. Omega 2 also regulates the expression of the $\omega-\varepsilon-\zeta$ operon involved in the regulation of the toxin-antitoxin system and the copS gene which regulates the number plasmid copies (de la Hoz et al., 2000; Zielenkiewicz and Ceglowski, 2005).

The parMRC, a type II partition system, was discovered in the multiresistant plasmid R1 in E. coli, the
pSK41 plasmid in $S$. aureus and on the pB171 virulence plasmid of E. coli (Ebersbach and Gerdes, 2005; Popp et al., 2010; Salje et al., 2010). The motor protein, designated ParM is an actin-like molecule with ATPase activity whereas the centromere-binding protein is referred to as ParR, and functions as a repressor of the segregation system. The centromeric locus, $\operatorname{par} C$ is composed of ten $11 \mathrm{bp}$ repeated nucleotide sequences located in two clusters. Each cluster is formed by five repeated-nucleotide sequences on each side at -10 and - 35 positions of the partition promoter region (Dam and Gerdes, 1994). ParM structure of pSK41 consists of ten $\alpha$-helices and $13 \beta$-strands. ParR of pB171 has two monomers and appears as an asymmetric unit since there is a short $\alpha$-helix in one monomer (Møller-Jensen et al., 2007; Popp et al., 2010).

The plasmid pBtoxis was isolated from Bacillus thuringiensis and contains the type III partition system. This system consists of TubZ, a tubulin-like protein that polymerizes into filamentous structures exploiting GTPase activity, and the centromere binding protein (TubR) acting on $t u b C$, the centromeric region which consists of 48 base-pairs arranged as four repeated sequences (Tang et al., 2006, 2007; Aylett and Löwe, 2012).

Two drugs phenoxybenzamine and octoclothepin suppressed the ATPase activity of ParA in Mycobacterium tuberculosis. Phenoxybenzamine decreased $50 \%$ of the ATPase activity at a concentration of $250 \mu \mathrm{M}$, while octoclothepin caused a $20 \%$ reduction in the ATPase activity at $25 \mu \mathrm{M}$ suggesting that these compounds may interact with the active site of the ParA molecule (Nisa et al., 2010). In silico homology modeling and molecular docking studies on ParF, a type Ib portioning protein, suggested that amentoflavone, hinokiflavone rutin, vicenin, silybin, and scutellarin had binding affinities towards the ATP active site (Al-Khayyat and Al-Dabbagh, 2015).

\section{Bacterial cytoskeleton}

Bacterial cytoskeleton elements could also serve as molecular targets to combat infections. These elements are subdivided into four classes: a) actin-like elements such as MreB, FtsA and ParM, b) Tubulin-like elements such as FtsZ, TubZ, RepX, ButbA and ButbB, c) Walker A ATPases designated WACA like MinD, ParA and ParF, and d) intermediate filaments like crescentin, CreS (Wickstead and Gull, 2011). 
MreB possesses an actin-like fold with ATPase activity (van den Ent et al., 2010). MreB with RodZ is responsible for the normal rod shape of bacteria such as E. coli and Caulobacter crescentus. MreB forms polymers, arranged spirally under the cytoplasmic membrane to allow for the expansion of the cell during the peptidoglycan synthesis (van den Ent et al., 2010). Iwai et al. (2002) found that a compound called S-(3,4-dichlorobenzyl)isothiourea exhibited a bactericidal effect on $E$. coli at a minimum inhibitory concentration of $3.1 \mu \mathrm{g} / \mathrm{ml}$. Spherical cells with variable sizes and without nucleus appeared when subjected to $10 \mu \mathrm{g} / \mathrm{ml}$ of this compound. Bean et al. (2009) suggested that this compound is a competitive inhibitor of ATP because its binds the highly conserved amino acids $\mathrm{Glu}^{131}$ and $\mathrm{Thr}^{158}$, occupying the binding regions of the phosphate groups.

According to Löwe and Amos (1998), filamentous temperature-sensitive mutant $Z$ denoted as Fts $Z$ is a dimer and is composed of two domains linked by an $\alpha$-helix which contains 23 residues. The $\mathrm{N}$-terminal portion of each monomer starts from residue 38 to 227 of the six $\beta$-strands arranged in a parallel manner with two or three $\alpha$-helices on each side. The $\mathrm{C}$ terminal portion starts from 228 to 356 , and contains four $\beta$-sheets in parallel and two $\alpha$-helices. GTP binds Fts $Z$ at the active site where GTP hydrolysis stimulates Fts Z molecules to polymerize and form a ring structure referred to as the $Z$-ring. The Z-ring recruits about ten other proteins to form the divisome complex that plays an important role in the formation of a septum in the middle of the cell, an essential step in cytokinesis (Errington et al., 2003; Adams and Errington, 2009).

Interfering with the FtsZ function, i.e., polymerization or the GTPase activity can seriously affect bacterial division and lead to cell death (Loose and Mitchison, 2014). Therefore, researchers are working to find inhibitors of FtsZ. Chemicals, and some natural compounds, which were proposed to have such action, are reviewed in detail in Vollmer (2006). Measurement of the level of FtsZ polymerization by light scattering assays showed that trans-cinammic acid has the highest level of inhibition among caffeic acid, naringenin, and eugenol. and 4-formyl cinnamic acid tested (Rastogi et al., 2008). Ten coumarins were tested in a study aiming to inhibit GTPase and polymer assembly functions of FtsZ. Scopoletin and daphnetin inhibited 50\% of GTPase activity at concentrations of 22 and $57 \mu \mathrm{M}$, respectively. Scopoletin inhibited $50 \%$ of Fts $Z$ polymerization at $41 \mu \mathrm{M}$ while daphentin required $72 \mu \mathrm{M}$ to achieve the same effect (Duggirala et al., 2014). Resveratrol possesses antibacterial activity resulting from: a) inhibition of the formation of the Z-ring since it reduces FtsZ genetic expression, b) changes in the morphology of the bacteria which become elongated as a consequence of a dysfunction in septum formation, c) chromosome damage, d) induction of the SOS response, and e) increased production of reactive oxygen species (Hwang and Lim, 2015).

Den Blaauween et al. (2014) suggested that other components of the divisome complex can also be exploited as targets in antimicrobial design, e.g., FtsEX, FtsW, FtsQLB, FtsA, ZipA and ClpP. Formation of the Z-ring requires FtsA to stabilize or anchor the ring into the cytoplasmic membrane and its C-terminal $\alpha$-helix is essential to perform this role. Loss of FtsZ activity results in a formation of long filament-like bacterial cells that cannot divide. The depletion of FtsA disturbs the process of septum formation, which causes elongated cells to appear with the assembly of several Z-rings, without the ability to complete the septum formation. Furthermore, the overall absence of FtsA leads to Z-ring assembly at different locations and cellular swelling, and lysis at the end (Pichoff and Lutkenhaus, 2005; Mura et al., 2017). The interaction interface between FtsAFtsZ may be used as a molecular target in the development of new antibacterials since FtsA interacts with the $\mathrm{Fts} Z$ and most other divisome components are recruited to the FtsZ ring through FtsA activity (Errington et al., 2003). Two compounds, namely, 3-(2-indolyl) piperidine and Indolo[2,3-a]quinolizin-7-one were tested and the results showed that they inhibited the interaction of the ZipA-FtsZ (Sutherland etal., 2003; Jennings et al., 2004). Paradis-Bleau et al. (2005) performed a study on P. aeruginosa and found that five short peptides, in concentrations ranging between $0.7-35 \mathrm{mM}$, disturbed $50 \%$ of the FtsA ATPase activity.

\section{Shikimate acid pathway}

The shikimate acid pathway (Fig. 1) is found in bacteria, parasites and plants but not in humans thus, it can be an excellent target for antimicrobials. This pathway is involved in the production of aromatic amino acids - tryptophan, tyrosine, and phenylalanine (Gasser et al., 1988; McDevitt et al., 2002). A library of 139 compounds was screened to identify inhibitors targeting 
Phosphoenolpyruvate + Erythrose-4-phosphate

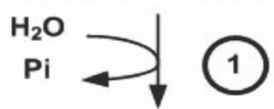

3-deoxy-D-arabino-heptulosonate 7-phosphate
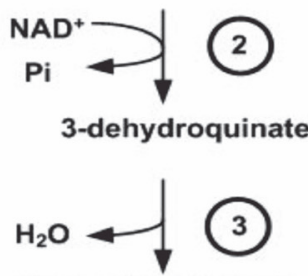

3-dehydroshikimate

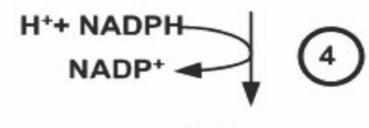

Shikimate

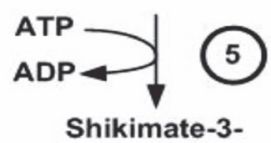

Shikimate-3-

phosphate

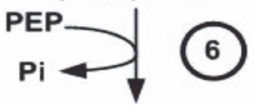

5-enolpyruvyl-shikimate-3-phosphate
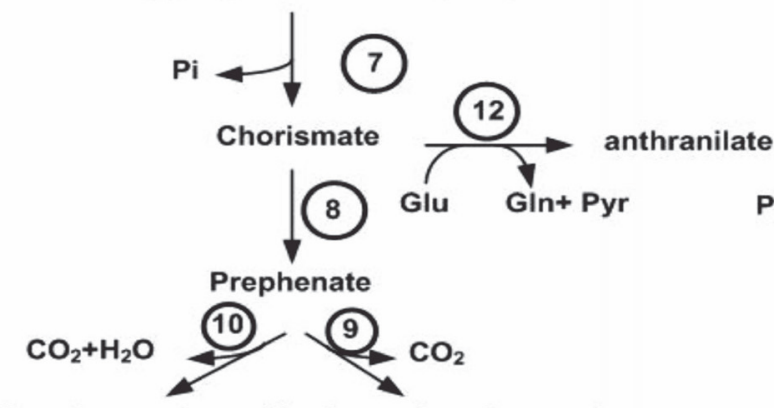

Phenylpyruvate 4-hydroxyphenylpyruvate

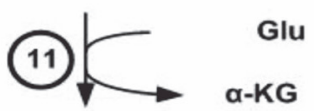

L- phenylalanine

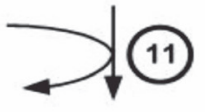

L- tyrosine
(13)

Pro PRPP
$\mathrm{N}-\left(5^{\prime}-\right.$ Phosphoribosyl)anthranilate

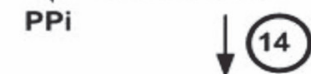

Enol-1-o-carboxyphenylamino1-deoxy-ribulose phosphate

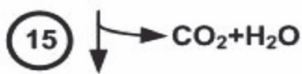

Indol-3-glycerol phosphate

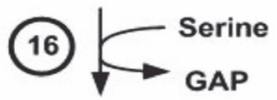

L- tryptophan

Fig. 1. Shikimate acid pathway. Abbreviations: Glu - glutamate, Gln - glutamine, Pyr - pyruvate, GAP - glyceraldehyde-3-phosphate, PRPP - 5-phosphoribosyl-1-pyrophosphate, PEP - phosphoenolpyruvate, $\alpha$-KG - $\alpha$-ketoglutarate; enzymes: (1) 3-deoxy-Darabino-heptulosonate 7-phosphate synthase, (2) 3-dehydroquinate synthase (3) 3-dehydroshikimate dehydratase (4) shikimate dehydrogenase (5) shikimate kinase (6) 5-enolpruvyl-shikimate-3-phosphate synthase (7) chorismate synthase (8) chorismate mutase (9) prephenate dehydrogenase (10) prephenate dehydratase (11) transaminase (12) anthranilate synthase (13) anthranilate phosphoribosyl transferase (14) phosphoribosyl-anthranilate isomerase (15) indole-3- glycerol phosphate synthase (16) tryptophan synthase. Reproduced with permission from BioMed Central Ltd. (Báez-Viveros et al. (2007)

3-dehydroquinate dehydratase, one of the enzymes of the shikimate pathway, in E. faecalis which catalyzes the formation of 3-dehydroshikimate. The flavonoids that inhibited the enzyme were: gossypin, rutin, homoorientin, luteolin-3', 7-diglucoside, luteolin-7-glucoside, and rhoi- folin. These flavonoids, however, did not affect the growth of the micro-organism while datiscetin and naringenin had this action. Marein, a chalconoid, inhibited the enzymatic activity of 3-dehydroquinate dehydratase and inhibited the growth of the pathogen. 7-hydroxy- 
coumarin and aesculin, belonging to coumarins, also inhibited the enzyme activity (Cheung et al., 2014).

Shikimate dehydrogenase is responsible for the conversion of 3-dehydroshikimate to shikimate. Five substances were able to inhibit shikimate dehydrogenase as shown in the study of Han et al. (2006). These were: a) curcumin, b) 3-(2-naphthyloxy)-4-oxo-2-(trifluoromethyl)4 H-chromen-7-yl 3-chlorobenzoate, c) butyl 2-\{[3-(2naphthyloxy)-4-oxo-2-(trifluoromethyl)-4H-chromen-7yl]oxy $\}$ propanoate, d) 2-(\{2-[(2-\{[2-(2,3-dimethylanilino)2-oxoethyl]sulfanyl \}-1,3-benzothiazol-6-yl)amino]-2oxoethyl \} sulfanyl)-N-(2-naphthyl)acetamide, and e) maesaquinone diacetate. These showed $50 \%$ inhibitory concentrations of $15.4,3.9,13.4,2.9$, and $3.5 \mu \mathrm{M}$, respectively. The first, second and fifth compounds also inhibited also the growth of Helicobacter pylori at minimum inhibitory concentrations of 16,16 , and $32 \mu \mathrm{g} / \mathrm{ml}$, respectively. Peek et al. (2014) screened about 5500 compounds to find inhibitors of shikimate dehydrogenase for $P$. putida, and found that two compounds, epigallocatechin gallate and epicatechin gallate, in concentrations of 3 and $3.7 \mu \mathrm{M}$, respectively, were able to inhibit $50 \%$ of the enzyme activity.

Shikimate kinase (SK) produces shikimate-3-phosphate by addition of a phosphate group to the shikimate. Two compounds, 1,3-methoxy-4-\{[2-(\{2-methoxy-4-[(4oxo-2-thioxo-1,3-thiazolidin-5-ylidene)methyl] phenoxy methyl) benzyl $]$ oxy $\}$ benzaldehyde, and 5-bromo-2-(5-\{[1(3,4-dichlorophenyl)-3,5-dioxo-4-pyrazolidinylidene] methyl\}-2-furyl)benzoic acid), in 5.5 and $6.4 \mu \mathrm{M}$ concentrations, respectively, inhibited $50 \%$ activity of the shikimate kinase of $H$. pylori (Han et al., 2007). A molecular docking study carried out on chorismate synthase of Listeria monocytogenes by Hossain et al. (2015) revealed four compounds with binding affinities to this enzyme: a) chromopyrrolic acid, b) N-(3-chlorophenyl)-Nmethyl-2-oxo-3-[(3,4,5-trimethyl-1H-pyrrol-2-yl) methyl] 2H-indole-5-sulfonamide, c) Salpha,10alpha,17beta)-17[(4-hydroxyphenyl)carbonyl] androsta-3,5-dien-3-carboxylic acid, and (d) N-[5-(1,1-dioxidoisothiazolidin-2-yl)-1Hindazol-3-yl]-2-(4-piperidin-1-yl phenyl) acetamide.

\section{Isoprenoid biosynthesis}

Isoprenoids constitute a large family of $35000 \mathrm{com}$ pounds involved in many functions such as cell wall biosynthesis, hormone processes of signaling and photo-

\section{Glyceraldehyde 3-phosphate}

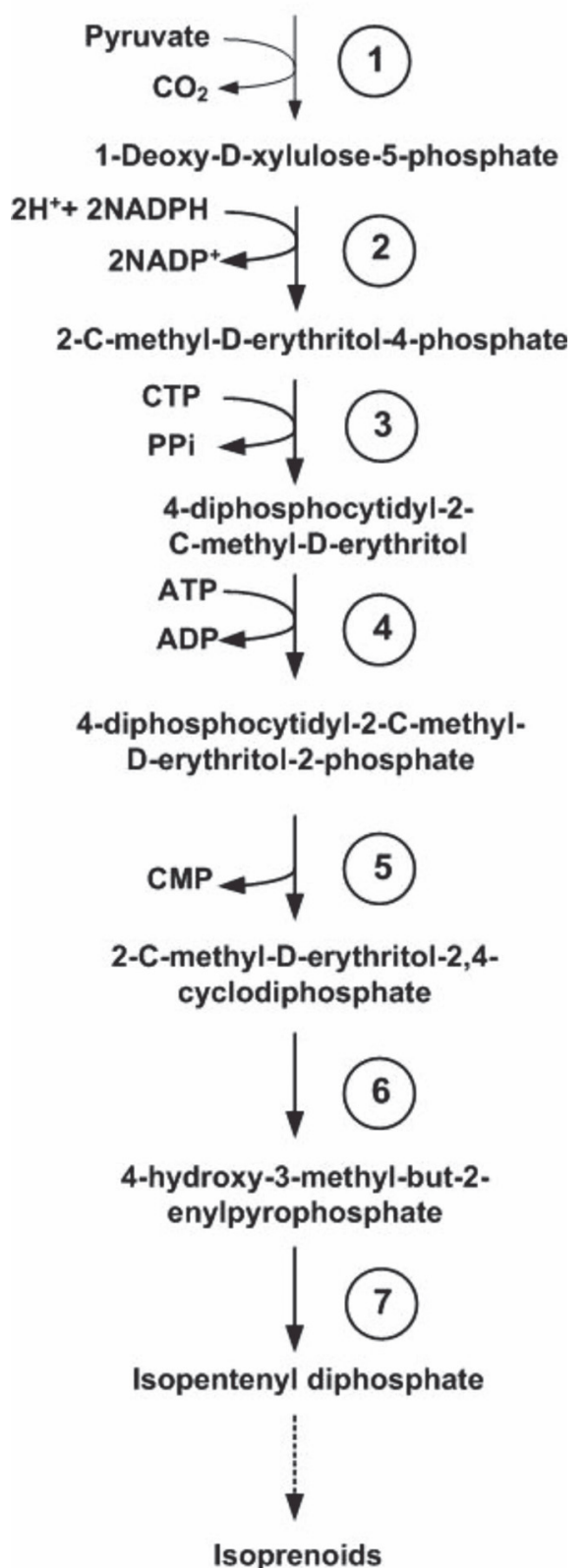

Fig. 2. Non-mevalonate pathway for isprenoid synthesis. Enzymes are (1) 1-deoxy-D-xylulose-5-phosphate synthase, DXS, (2) 1-deoxy-D-xylulose-5-phosphate reductoisomerase, IspC, (3) 4-diphosphocytidyl-2-C-methyl-P-erythritol synthase, IspD, (4) 4-diphosphocytidyl-2-C-methyl-P-erythritol kinase, IspE, (5) 2-C-methyl-D-erythritol 2,4-cyclodiphosphate synthase, IspF, (6) 4-hydroxy-3-methyl-but-2-enylpyrophosphate synthase, IspG, (7) 4-hydroxy-3-methyl-but-2-enylpyrophosphate synthase, IspH. Modified with permission of PLoS ONE (Tidten-Luksch et al., 2012)

synthesis, electron transport, transcription, and posttranslational events, synthesis of glycoprotein, breakdown of protein and meiotic division (Hunter, 2007; Heuston et al., 2012). The synthesis of isoprenoids takes 
place via two metabolic pathways, the mevalonate pathway and the non-mevalonate pathway, also known as a mevalonate-independent pathway (Lange et al., 2000). The mevalonate pathway starts which the condensation of acetyl-CoA and acetoacetyl-CoA to form 3-hydroxy-3methylglutaryl-CoA which is converted to mevalonate via a reduction step. It is present in eukaryotes including plants, fungi, mammals, archaea and some Gram-positive bacteria like $S$. aeurus, $S$. pneumoniae, and $E$. faecalis (Goldstein and Brown, 1990; Hunter, 2007; Wilding et al., 2000). The independent pathway (Fig. 2) starts with the reaction of glyceraldehyde-3-phosphate with pyruvate to form 1-deoxyxylulose-5-phosphate. This pathway is present in many Gram-negative bacteria and some Gram-positive bacteria (Lange et al., 2000).

Fosmidomycin can inhibit the enzyme 1-deoxy-D-xylulose-5-phosphate reductoisomerase, designated as IspC in malarial parasites (Jomaa et al., 1999) since it contains a phosphonate group instead of the phosphate group in 1-deoxy-D-xylulose-5-phosphate, the substrate of this enzyme. Structural analogs were investigated by modifications of the phosphonate and hydroxamate groups or substitution of the aliphatic chain with an aromatic ring (Lienau et al., 2015). The enzyme 4-diphosphocytidyl-2-C-methyl-D-erythritol synthase, IspD catalyzes the conversion of 2-C-methyl-D-erythritol-4-phosphate to 4-diphosphocytidyl-2-C-methyl-D-erythritol by the addition of a phosphate group from cytidine-3-phosphate (CTP). Natural products called pseudilins were found to inhibit this enzyme via binding the CTP site, in experiments carried on Plasmodium vivax (Kunfermann et al., 2014).

\section{Conclusions}

Searching databases for experimental compounds and natural products can assist in identifying possible inhibitors of these new molecular targets in bacteria. Elements of cytoskeleton and metabolic pathways that are unique to bacteria could be a target for new antibiotics to overcome resistance issues. The new antibiotic discovery process should be accompanied by a study of pharmacokinetics and pharmacodynamics, together with animal studies, to explore the usefulness and adverse effects of the compounds proposed to be used in antimicrobial chemotherapy.

\section{Acknowledgments}

The author would (also) like to thank BioMed Central Ltd., the original publisher of Báez-Viveros et al., Microbial Cell Factories $2007 ; 6: 30$, for their permission to reproduce Fig. 2 and PLoS One for the permission to reproduce Fig. 1 that appeared in Tidten-Luksch et al., 2014, PLoS One 7(4): e35792.

\section{References}

Adams D.W., Errington J. (2009) Bacterial cell division: assembly, maintenance and disassembly of the $Z$ ring. Nat. Rev. Microbiol. 7(9): 642-653.

Al-Khayyat M.Z., Al-Dabbagh AG. (2015) Homology modeling and docking studies of a plasmid partition protein, ParF: Flavonoids as anti-plasmid agents. J. Comput. Methods Mol. Des. 5(3): 25-32.

Asad S., Opal S.M. (2008) Bench-to-bedside review: Quorum sensing and the role of cello-cell communication during invasive bacterial infection. Critical Care 12(6): 236.

Austin S., Sengupta M. (2011) Prevalence and significance of plasmid maintenance functions in the virulence plasmids of pathogenic bacteria. Infect. Immun. 79(7): 2502-2509.

Aylett C.H.S., Löwe J. (2012) Superstructure of the centromeric complex of TubZRC plasmid partitioning systems. Proc. Natl. Acad. Sci. USA 109: 16522-16527.

Báez-Viveros J.L., Flores N., Juárez K., Castillo-Espańa P., Bolivar F., Gosset G. (2007) Metabolic transcription analysis of engineered Escherichia coli strains that overproduce $L$ phenylalanine. Microb. Cell Fact. 6(1): 30.

Barnard A.M.L., Salmond G.P.C. (2007) Quorum sensing in Erwinia species. Anal. Bioanal. Chem. 387: 415-423.

Baxter J.C., Funnell B.E. (2014) Plasmid partition mechanisms. Microbiol. Spectrum 2(6): PLAS-0023-2014.

Bean G.J., Flickinger S.T., Westler W.M., McCully M.E., Sept D., Weibel D.B., Amann K.J. (2009) A22 disrupts the bacterial actin cytoskeleton by directly binding and inducing a low-affinity state in MreB. Biochemistry 48(22): 4852-4857.

Behera B., Mathur P. (2011) High levels of antimicrobial resistance at a tertiary trauma care centre of India. Indian J. Med. Res. 133(3): 343-345.

Bush K. (2013) Proliferation and significance of clinically relevant B-lactamases. Ann. NY Acad Sci.1277: 84-90.

Cao B., Xiao F., Xing D., Hu X. (2018) Polyprodrug antimicrobials: Remarkable membrane damage and concurrent drug release to combat antibiotic resistance of methicillinresistant Staphylococcus aureus. Small 1802008.

Ceglowski P., Boitsov A., Chai S., Alonso J.C.(1993) Analysis of the stabilization system of pSM19035-derived plasmid pBT233 in Bacillus subtilis. Gene 136: 1-12.

Cheung V.W.N, Xue B., Hernandez-Valladares M., Go M.K., Tung A., Aguda A.H., Robinson R.C., Yew W.S. (2014) Identification of polyketide inhibitors targeting 3-dehydroquinate dehydratase in the shikimate pathway of Enterococcus faecalis. PLoS ONE 9(7): e103598. 
Coulthurst S.J., Williamson N.R., Harris A.K., Spring D.R., Salmond G.P. (2006) Metabolic and regulatory engineering of Serratia marcescens: mimicking phage-mediated horizontal acquisition of antibiotic biosynthesis and quorum-sensing capacities. Microbiology 152: 1899-1911.

Culp E., Wright G.D. (2017) Bacterial proteases, untapped antimicrobial drug targets. J. Antibiotics 70: 366-377.

Dam M., Gerdes K. (1994) Partitioning of plasmid R1. Ten direct repeats flanking the parA promoter constitute a centromere-like partition site parC, that expresses incompatibility. J. Mol. Biol. 236: 1289-1298.

de la Hoz A.B., Ayora S., Sitkiewicz I., Fernandez S., Pankiewicz R., Alonso J.C., Ceglowski P. (2000) Plasmid copynumber control and better-than-random segregation genes of pSM19035 share a common regulator. Proc. Natl. Acad. Sci. USA 97: 728-733.

Den Blaauwen T., Andreu J.M., Monasterio O. (2014) Bacterial cell division proteins as antibiotic targets. Bioorg. Chem. 55: 27-38.

Duggirala S., Nankar R.P., Rajendran S., Doble M. (2014) Phytochemicals as inhibitors of bacterial cell division protein FtsZ: coumarins are promising candidates. Appl. Biochem. Biotechnol. 174: 283-296.

Ebersbach G., Gerdes K. (2005) Plasmid segregation mechanisms. Annu. Rev. Genet. 39: 453-479.

Errington J., Daniel R.A., Scheffers D.-J. (2003) Cytokinesis in bacteria. Microbiol. Mol. Biol. Rev. 67: 52-65.

Fuqua C., Greenberg E.P. (2002) Listening in on bacteria: acyl-homoserine lactone signalling. Nat. Rev. Mol. Cell Biol. 3(9): 685-695.

Gasser C.S., Winter J.A., Hironaka C.M., Shah D.M. (1988) Structure, expression and evolution of 5-enolpyruvyl shikimate-3-phosphate synthase genes of petunia and tomato. J. Biol. Chem. 263(9): 4280-4289.

Gerdes K., Moller-Jensen J., Jensen R.B. (2000) Plasmid and chromosome partitioning: surprises from phylogeny. Mol. Microbiol. 37: 455-466.

Goldstein B.P. (2014) Resistance to rifampicin: a review. J. Antibiot. 67: 625-630.

Goldstein J.L., Brown M.S. (1990) Regulation of the mevalonate pathway. Nature 343: 425-430.

Han C., Wang L., Yu K., Chen L., Hu L., Chen K., Jiang H., Shen X. (2006) Biochemical characterization and inhibitor discovery of shikimate dehydrogenase from Helicobacter pylori. FEBS J. 273(20): 4682-4692.

Han C., Zhang J., Chen L., Chen K., Shen X., Jiang H.(2007) Discovery of Helicobacter pylori shikimate kinase inhibitors. bioassay and molecular modeling. Bioorg. Med. Chem. 15(2): 656-662.

Hart C.A., Kariuki S. (1998) Antimicrobial resistance in developing countries. BMJ 317: 647-650.

Heuston S., Begley M., Gahan C.G., Hill C. (2012) Isoprenoid biosynthesis in bacterial pathogens. Microbiology 158: 1389-1401.

Hossain M.M., Roy P.K., Jannatul Mosnaz A.T.M., Shakil S.K., Hasan M.M., Prodhan S.H. (2015) Structural analysis and molecular docking of potential ligands with chorismate synthase of Listeria monocytogenes: A novel antibacterial drug target. Indian J. Bioch. Bioph. 52: 45-59.

Hunter W.N. (2007) The non-mevalonate pathway of isoprenoid precursor biosynthesis. J. Biol. Chem. 282(30): 21573-21577.

Hussein S.S., Abeed A.K., Tktok N.K. (2017) Study of effect of some antibiotics on Pseudomonas aeruginosa and Staphylococcus aureus isolated from burns and wounds infections. Babylon Uni. J. 25(1): 69-80.

Hwang D., Lim Y.-H. (2015) Resveratrol antibacterial activity against Escherichia coli is mediated by Z-ring formation inhibition via suppression of Fts $Z$ expression. Nat. Sci. Rep. 5: 10029.

Iwai N., Nagai K., Wachi M. (2002) Novel S-benzylisothiourea compound that induces spherical cells in Escherichia coli probably by acting on a rod-shape-determining protein(s) other than penicillin-binding protein 2. Biosci. Biotechnol. Biochem. 66(12): 2658-2662.

Jennings L.D., Foreman K.W., Rush T.S., Tsao D.H., Mosyak L., Li Y., Sukhdeo M.N., Ding W., Dushin E.G., Kenny C.H., Moghazeh S.L., et al. (2004) Design and synthesis of indolo[2,3-a]quinolizin-7-one inhibitors of the ZipA-FtsZ interaction. Bioorg. Med. Chem. Lett. 14(6): 1427-1431.

Jomaa H., Wiesner J., Sanderbrand S., Altincicek B., Weidemeyer C., Hintz M., Turbachova I., Eberl M., Zeidler J., Lichtenthaler H.K. et al. (1999) Inhibitors of the nonmevalonate pathway of isoprenoid biosynthesis as antimalarial drugs. Science 285(5433): 1573-1576.

Joshi J.R., Khazanov N., Senderowitz H., Burdman S., Lipsky A., Yedidia I. (2016) Plant phenolic volatiles inhibit quorum sensing in pectobacteria and reduce their virulence by potential binding to ExpI and ExpR proteins. Nat. Sci. Rep. 6: 38126 .

Kunfermann A., Witschel M., Illarionov B., Martin R., Rottmann M., Höffken W., Seet M., Eisenreich W., Knölker H.J., Fischer M. et al. (2014) Pseudilins: halogenated, allosteric inhibitors of the non mevalonate pathway enzyme IspD. Angewandte Chemie 53(8): 2235-2239.

Lange B.M., Rujan T., Martin W., Croteau R. (2000) Isoprenoid biosynthesis: the evolution of two ancient and distinct pathways across genomes. Proc. Natl. Acad. Sci. 97(24): 13172-13177.

Larsen R.A., Cusumano C., Fujioka A., Lim-Fong G., Patterson P., Pogliano J. (2007) Treadmilling of a prokaryotic tubulin-like protein, TubZ, required for plasmid stability in Bacillus thuringiensis. Genes Dev. 21: 1340-1352.

Lienau C., Konzuch S., Gräwert T., Illarionov B., Bacher A., Fischer M., Tanaka N., Kurz T. (2015) Inhibition of the nonmevalonate Isoprenoid pathway by reverse hydroxamate analogues of fosmidomycin. Proc. Chem. 14: 108-116.

Liu H., Coulthurst S.J., Pritchard L., Hedley P.E., Ravensdale M., Humphris S., Burr T., Takle G., Brurberg M-B., Birch P.R.J., et al. (2008) Quorum sensing coordinates brute force and stealth modes of infection in the plant pathogen Pectobacterium atrosepticum. PLoS Pathog. 4(6): e1000093. 
Loose M., Mitchison T.J. (2014) The bacterial cell division proteins Fts $A$ and Fts $Z$ self-organize into dynamic cytoskeletal patterns. Nat. Cell. Biol. 16(1): 38-46.

Löwe J., Amos L.A. (1998) Crystal structure of the bacterial cell-division protein FtsZ. Nature 391: 203-206.

McDevitt D., Payne D.J., Holmes D.J., Rosenberg M. (2002) Novel targets for the future development of antibacterial agents. J. Appl. Microbiol. 92: 28S-34S.

Miller M.B., Bassler B.L. (2001) Quorum sensing in bacteria. Annu. Rev. Microbiol. 55: 165-199.

Moghaddam M.M., Khodi S., Mirhosseini A. (2014) Quorum sensing in bacteria and a glance on Pseudomonas aeruginosa. Clin. Microbiol. 3: 156.

Mřller-Jensen J., Ringgaard S., Mercogliano C.P., Gerdes K., Lowe J. (2007) Structural analysis of the ParR/parC plasmid partition complex. EMBO J. 26: 4413-4422.

Morgan D.J., Okeke I.N., Laxminarayan R., Perencevich E.N., Weisenberg S. (2011) Nonprescription antimicrobial use worldwide: a systematic review. Lancet Infect. Dis. 11(9): 692-701.

Mura A., Fadda D., Perez A.J., Danforth M.L., Musu D., Rico A.I., Krupka M., Denapaite D., Tsui H.-C.T., Winkler M.E. et al. (2017) Roles of the essential protein FtsA in cell growth and division in Streptococcus pneumoniae. J. Bacteriol. 199(3): e00608-e00616.

Nisa S., Blokpoel M.C.J., Robertson B.D., Tyndall J.D.A., Lun S., Bishai W. R., O'Toole R. (2010) Targeting the chromosome partitioning protein ParA in tuberculosis drug discovery. J. Antimicrob. Chemother. 65: 2347-2358.

Paradis-Bleau C., Sanschagrin F., Levesque R.C. (2005) Peptide inhibitors of the essential cell division protein FtsA. Prot. Engneer. Design Select. 18(2): 85-91.

Peek J., Shi T., Christendat D. (2014) Identification of novel polyphenolic inhibitors of shikimate dehydrogenase (AroE). J. Biomol. Screen. 19(7): 1090-1098.

Pichoff S., Lutkenhaus J. (2005) Tethering the $Z$ ring to the membrane through a conserved membrane targeting sequence in FtsA. Mol. Microbiol. 55(6): 1722-1734.

Piddock L.J.V. (2006) Clinically relevant chromosomally encoded multidrug resistance efflux pumps in bacteria. Clin. Microbiol. Rev. 19(2): 382-402.

Popp D., Xu W., Narita A., Brzoska A.J., Skurray R.A, Firth N., Goshdastider U., Maéda Y., Robinson R.C., Schumacher M.A. (2010) Structure and filament dynamics of the pSK41 actin-like ParM protein: implications for plasmid DNA segregation. J. Biol. Chem. 285(13): 10130-10140.

Pratto F., Cicek A., Weihofen W.A., Lurz R., Saenger W., Alonso J.C. (2008) Streptococcus pyogenes pSM19035 requires dynamic assembly of ATP-bound ParA and ParB on parS DNA during plasmid segregation. Nucl. Acids Res. 36: 3676-3689.

Ramirez M.S., Tolmasky M.E. (2010) Aminoglycoside modifying enzymes. Drug Resist. Updat. 13(6): 151-171.

Rastogi N., Domadia P., Shtty S., Dasgupta D. (2008) Screening of natural phenolic compounds for potential inhibit bac- terial cell division protein FtsZ. Indian J. Exp. Biol. 46: 783-787.

Salje J., Pand G., Löwe J. (2010) The ParMRC system: molecular mechanisms of plasmid segregation by actin-like filaments. Nat. Rev. Microbiol. 8: 683-692.

Sergueev K., Dabrazhynetskaya A., Austin S. (2005) Plasmid partition system of the P1 par family from the $p W R 100$ virulence plasmid of Shigella flexneri. J. Bacteriol. 187: 3369-3373.

Song Z., Kong K.F., Wu H., Maricic N., Ramalingam B., Priestap H., Quirke J.M.E., Hřiby N., Mathee K. (2010) Panax ginseng has anti-infective activity against opportunistic pathogen Pseudomonas aeruginosa by inhibiting quorum sensing, a bacterial communication process critical for establishing infection. Phytomedicine 17: 1040-1046.

Sutherland A.G., Alvarez J., Ding W., Foreman K.W., Kenny C.H., Labthavikul P., Mosyak L., Petersen P.J., Rush T.S., Ruzin A. et al. (2003) Structure-based design of carboxybiphenylindole inhibitors of the ZipA-FtsZ interaction. Org. Biomol. Chem. 1(23): 4138-4140.

Tang M., Bideshi D.K., Park H-W., Federici B.A. (2007) Iteronbinding ORF157 and FtsZ-like ORF156 proteins encoded by pBtoxis play a role in its replication in Bacillus thuringiensis subsp. israelensis. J. Bacteriol. 189: 8053-8058.

Tang M., Bideshi D.K., Park H.W., Federici B.A. (2006) Minireplicon from pBtoxis of Bacillus thuringiensis subsp. israelensis. Appl. Environ. Microbiol. 72: 6948-6954.

Thomson N.R., Crow M.A., McGowan S.J., Cox A., Salmond G.P. (2000) Biosynthesis of carbapenem antibiotic and prodigiosin pigment in Serratia is under quorum sensing control. Mol. Microbiol. 36: 539-556.

Tidten-Luksch N., Grimaldi R., Torrie L.S., Frearson J.A., Hunter W.N., Brenk R. (2012) IspE inhibitors identified by a combination of in silico and in vitro high-throughput screening. PLoS ONE 7(4): e35792.

van den Ent F., Johnson C.M., Persons L., de Boer P., Löwe, J. (2010) Bacterial actin MreB assembles in complex with cell shape protein RodZ. EMBO J. 29(6): 1081-1090

Van Houdt R., Michiels C.W. (2005) Role of bacterial cell surface structures in Escherichia coli biofilm formation. Res. Microbiol. 156: 626-633.

Vandeputte O.M., Kiendrebeogo M., Rasamiravaka T., Stévigny C., Duez P., Rajaonson S., Diallo B., Mol A., Baucher M., El Jaziri M. (2011) The flavanone naringenin reduces the production of quorum sensing-controlled virulence factors in Pseudomonas aeruginosa PAO1. J. Microbiology. 157: 2120-2132.

Vollmer W. (2006) The prokaryotic cytoskeleton: a putative target for inhibitors and antibiotics? Appl. Microbiol. Biotechnol. 73(1): 37-47.

Wickstead B., Gull K. (2011) The evolution of the cytoskeleton. J. Cell. Biol. 194(4): 513-525.

Wilding E.I., Brown J.R., Bryant A.P., Chalker A.F., Holmes D.J., Ingraham K.A., Iordanescu S., So C.Y., Rosenberg M., Gwynn M.N. (2000) Identification, evolution, and essentiality of the mevalonate pathway for isopentenyl diphosphate 
biosynthesis in gram-positive cocci. J. Bacteriol. 182(15): 4319-4327.

Wilson D.N. (2014) Ribosome-targeting antibiotics and mechanisms of bacterial resistance. Nat. Rev. Microbiol. 12(1): $35-48$.

Zielenkiewicz U., Ceglowski P. (2005) The toxin-antitoxin system of the streptococcal plasmid pSM19035. J. Bacteriol. 187: 6094-6105. 